\title{
Caracterización cineantropométrica de la población que acude a la Clínica Las Américas. Estudio observacional, retrospectivo
}

\author{
Cineanthropometric characterization of the population attending the Las \\ Americas Clinic. An observational, retrospective study
}

Rosero RJ, ${ }^{1}$ Polanco JP, Jaramillo $A,{ }^{3}$ Cossio I, ${ }^{4}$ Gómez AM, ${ }^{5}$ Atehortúa AM, ${ }^{6}$ Loaiza $S,{ }^{7}$ Palacio JI. ${ }^{8}$

${ }^{1}$ Médico Internista y Endocrinólogo, COD2, Clínica Las Américas, Medellín, Colombia.

${ }^{2}$ Médico Internista y Epidemiólogo, COD2, Clínica Las Américas, Medellín, Colombia.

${ }^{3}$ Médica Pediatra y Endocrinóloga, COD2, Clínica Las Américas, Medellín, Colombia.

${ }^{4}$ Médico, Universidad CES, Medellín, Colombia.

${ }^{5}$ Médico, Universidad de La Sabana, Chía, Colombia.

${ }^{6}$ Profesional en Sistemas de Información en Salud, Coordinadora de investigación clínica, Clínica Las Américas, Medellín, Colombia.

${ }^{7}$ Médico Máster en Epidemiología, Clínica Las Américas, Medellín, Colombia.

${ }^{8}$ Médico Deportólogo, COD2, Clínica Las Américas, Medellín, Colombia.

Correspondencia: COD2@clinicalasamericas.com.co

Fecha de recepción: 27/06/2018

Fecha de aceptación: 22/11/2018

\section{Resumen}

Introducción: El índice de masa corporal (IMC) es un parámetro simple de peso por altura que se usa comúnmente para clasificar bajo peso, sobrepeso y obesidad en adultos. Éste no permite distinguir entre el peso por músculo o por tejido graso. Como resultado, la relación entre el IMC y el contenido de grasa corporal varía de acuerdo con la constitución y la proporción corporal, demostrándose repetidamente que un IMC determinado no se ajusta al grado de riesgo o enfermedad entre diferentes poblaciones ${ }^{(1,2)}$.

Objetivo: Determinar cuál es el biotipo por cineantropometría y su riesgo cardiovascular asociado por IMC, de la población que acude al Centro de Obesidad, Dismetabolismo y Deporte (COD2), de la Clínica Las Américas de la ciudad de Medellín.

Materiales y métodos: Realizamos un estudio observacional retrospectivo de la población mayor de 18 años que asistió al COD2 entre los meses de julio y diciembre de 2017, determinando índices, antropometrías y composición corporal; se correlacionaron estos valores entre los diferentes grados de obesidad.

Resultados: En la población en estudio, el $41 \%$ de la población masculina y el 55\% de la población femenina con IMC normal o sobrepeso, presentó porcentaje graso corporal (PGC) en rangos de obesidad, lo cual se correlacionó con alteraciones de perímetro abdominal, índice cintura-cadera y grasa visceral elevados estadísticamente significativo $(\mathrm{p}<0,001)$.

Conclusiones: Existe un alto número de individuos con índices antropométricos anormales, con peso normal o sobrepeso, a los cuales otras herramientas de medición más individualizadas, como composición corporal, podrán favorecer una oportuna intervención. Se necesitan estudios poblacionales de mayor número para poder determinar los diferentes fenotipos en Colombia y su impacto en la salud.

Palabras clave: Obesidad, composición corporal, riesgo cardiovascular.

\section{Abstract}

Introduction: The Body Mass Index (BMI) is a simple weight by height parameter that is commonly used to classify underweight, overweight and obese in adults. This does not allow to distinguish between the muscle mass or fat mass. As a result, the relationship between BMI and body fat content varies according to constitution and body proportion, demonstrating repeatedly that a certain BMI does not adjust to the degree of risk or disease among different population $\boldsymbol{s}^{(1,2)}$.

objective: Characterize the cineanthropometric biotype and the cardiovascular risk by anthropometry according to BMI of the population attending the Obesity, Dismetabolism and Sports Center (COD2), of the Las Americas Clinic in the city of Medellín.

Material and methods: We conducted a retrospective observational study of the population over 18 years old, who at- 
tended COD2 between the months of July to December 2017, determining anthropometry index and body composition. These values were correlated between the different degrees of obesity.

Results: Among the study population, $41 \%$ of the male population and $55 \%$ of the female population with normal weight or overweight by BMI, presented body fat percentage (BFP) in obesity ranges. This was correlated with statistically significant alterations in waist circumference, waist-hip index and visceral fat $(p<0.001)$.

Conclusions: There is a high number of individuals with abnormal anthropometric indexes, with normal weight or overweight, to which other more individualized measurement tools such as body composition, may favor an early intervention. Larger population study is needed to determine the different phenotypes in Colombia and their impact on health.

Key words: Obesity, body composition, cardiovascular risk.

\section{Introducción}

La obesidad se define como el aumento de tejido adiposo en número y tamaño, que lleva a disfunción desfavorable sobre la salud y genera una mayor morbilidad y mortalidad ${ }^{(2)}$. La prevalencia de la obesidad sigue creciendo globalmente ${ }^{(1)}$ y su herramienta de identificación, IMC, es un parámetro simple de peso por altura que se usa comúnmente para clasificar bajo peso, sobrepeso y obesidad en adultos. Esta herramienta no distingue entre el peso asociado con el músculo o con la grasa. Como resultado, la relación entre el IMC y el contenido de grasa corporal varía de acuerdo con la constitución y la proporción corporal, y se ha demostrado repetidamente que un IMC determinado puede no corresponder al mismo grado de obesidad entre las poblaciones ${ }^{(2,3)}$.

La Organización para la Cooperación y Desarrollo Económico (OCDE), en su reporte del 2017, muestra una prevalencia de obesidad global de $19,5 \%{ }^{(4)} \mathrm{y}$, además de esto, el crecimiento exponencial de las enfermedades crónicas no transmisibles (hipertensión, dislipidemia, diabetes, entre otras). En Colombia, según la encuesta nacional de la situación nutricional (ESIN 2015) ${ }^{(5)}$, la prevalencia de sobrepeso es de $56,4 \%$ y $18,7 \%$ de obesidad, lo que favorece el incremento de las comorbilidades asociadas más frecuentes como son dislipidemia, hipertensión y diabetes ${ }^{(6,7)}$, así como enfermedades crónicas no transmisibles como riesgos cardiovasculares, diabetes tipo 2, hígado graso, cáncer, enfermedades obstructivas del sueño $0^{(1)}$, con la subsecuente disminución en la expectativa de vida ${ }^{(2,3)}$.

A pesar de que la obesidad es una enfermedad crónica, su diagnóstico y tratamiento se descuida con frecuencia y no se piensa en ella como una condición potencialmente mortal ${ }^{(7,8)}$. Al ver la otra cara de la moneda, cuando la obesidad no es la protagonista principal, se puede observar cómo las cifras de las enfermedades crónicas siguen incrementándose sin una relación directa con la obesidad. Esto mismo evidenciaron Wildman y colaboradores en el análisis de la población participante en la encuesta Nacional de Examen de Salud y Nutrición (NHANES), según fenotipos y riesgo cardiometabólico, donde se observa que el $23,5 \%$ y $48,7 \%$ de la población presenta alteraciones metabólicas, con IMC $<24,9 \mathrm{~kg} / \mathrm{m}^{2}$ y $>25-<29,9 \mathrm{~kg} /$ $\mathrm{m}^{2}$, respectivamente $\mathrm{e}^{(9,10)}$.

Aunque el exceso de adiposidad y no el exceso de peso corporal es el verdadero culpable de las complicaciones asociadas a la obesidad, los estudios que examinan el efecto de los riesgos de salud asociados a la obesidad en los que se mide la adiposidad son menos frecuentes de lo desea$\mathrm{do}^{(11)}$. El PGC se puede medir mediante diferentes técnicas, que abarcan mediciones desde pliegue de la piel (con baja sensibilidad), hasta imágenes por resonancia magnética (de alta especificidad) ${ }^{(12)}$. Otros métodos usados frecuentemente para determinar el PGC incluyen el análisis de impedancia bioeléctrica (BIA) y la absorciometría de rayos X de energía dual (DEXA) y entre los métodos más precisos y reproducibles se incluyen el pesaje subacuático y la pletismografía por desplazamiento del aire (ADP) ${ }^{(12-14)}$.

Cuando la determinación del PGC no está disponible, el IMC es la medida sustituta de obesidad más frecuentemente utilizada, sin que esto discrimine adiposidad. Sin embargo, el IMC, aunque fácil de calcular, exhibe notables imprecisiones que no reflejan precisamente la grasa corporal, los cambios en la composición corporal que tienen lugar en los diferentes periodos de la vida y las diferencias según el género ${ }^{(15,16)}$.

Se han publicado varias ecuaciones de predicción que tienen en cuenta el sexo y/o la edad para convertir el peso y la estatura a grasa corporal, los cuales han mostrado razonable eficacia para superar el problema mencionado, pero se han derivado de muestras pequeñas o de métodos imprecisos para medición de la composición corporal ${ }^{(17-19)}$.

Lo anterior posiblemente evoque la pregunta ¿por qué es crucial disponer de un estimador preciso de PGC? A esta pregunta ya se tienen diferentes respuestas como la de Linge y colaboradores, que logran establecer, según distribución y tamaño, la relación con desenlaces cardiometabólicos ${ }^{(20)}$. Por tanto, el objetivo del presente estudio es caracterizar la población que acude a nuestro centro para identificar biotopos cineantropométricos que nos permitan individualizar efectivamente los programas de intervención del paciente.

\section{Materiales y métodos}

Realizamos un estudio descriptivo, retrospectivo de la población mayor de 18 años, que asistió a la unidad médica entre los meses de julio y diciembre de 2017. Pertenecientes al régimen contributivo del sistema de seguridad social y salud de Colombia, las variables sociodemográficas fueron: sexo (masculino y femenino); edad ( $>18$ años); en cuanto a las varia- 
bles antropométricas: IMC, perímetro abdominal (PA) (como determinante de obesidad central $>92 \mathrm{~cm}$ en los hombres y $>84 \mathrm{~cm}$ en las mujeres) ${ }^{(21)}$; perímetro de cuello (PCu) elevada

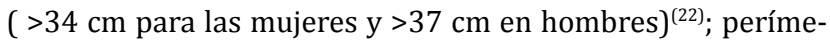
tro de cadera (PCa), utilizando técnica estandarizada según la $\mathrm{OMS}^{(23)}$. Se midieron las circunferencias de la cintura y la cadera con un margen de $1 \mathrm{~mm}$ en la cintura entre la costilla más baja y la cresta ilíaca, con el paciente en bipedestación al final de la espiración suave. Las caderas se midieron en el trocante mayor y el perímetro de cuello en la zona media. En los hombres con prominencia laríngea (manzana de Adán) se midió justo debajo de ésta. De igual forma, el PGC, grasa visceral (GV) contenido músculo-esquelético (MME), IMC, masa grasa corporal (MGC), masa libre de grasa (MLG) fueron determinados por impedanciometría en equipo InBody 770, en decúbito supino, sin ropa y con vejiga vacía.

\section{Estadística}

Dentro del plan de análisis se determinaron frecuencias absolutas y relativas para las variables categóricas; para las variables cuantitativas se utilizaron medidas de tendencia central (media o mediana) y/o dispersión (desviación estándar o percentiles) según la distribución de los datos. En el caso de las variables cuantitativas, las diferencias estadísticamente significativas entre los grupos de las cinco categorías del IMC se estipularon empleando ANOVA de una vía o la prueba de Kruskal-Wallis según la distribución normal o no de los datos; para las variables cualitativas se empleó la prueba de Chi cuadrado. Se establecieron diferencias estadísticamente significativas con un valor de p inferior a 0,05. Todo el análisis y procesamiento de la información se realizó con los paquetes estadísticos del programa $\mathrm{R}$ versión 3.4.3. (The R Foundation for Statistical Computing).

\section{Consideraciones éticas}

Esta investigación cumple con lo establecido en la Resolución 8430 de 1993 del Ministerio de Salud de la República de Colombia y puede ser clasificada como Investigación sin riesgo. Durante la investigación se garantizará la confidencialidad de la información obtenida a partir de las historias clínicas de los pacientes y los investigadores se comprometen a no divulgar en sus publicaciones cualquier información que permita la identificación de éstos. Se respetará el derecho a la no participación de aquellos pacientes que en el consentimiento informado de ingreso a la Clínica hayan denegado el campo específico en el cual autorizan el uso de su información clínica para estudios de investigación. Cuando se estime necesario, los pacientes podrán solicitar la información que consideren necesaria con relación a los propósitos, procedimientos, instrumentos de recopilación de datos y divulgación de los resultados del estudio.

\section{Resultados}

Se incluyeron un total de 257 individuos, que asistieron al centro médico COD2 con motivo de consulta por deseo de disminución de peso. Para el análisis se separaron los grupos por sexo, y se obtuvieron 97 hombres y 160 mujeres. A cada grupo se le dividió según IMC en normopeso $\left(19-24,9 \mathrm{~kg} / \mathrm{m}^{2}\right)$, sobrepeso $\left(25-29,9 \mathrm{~kg} / \mathrm{m}^{2}\right)$ y obesidad $\left(>30 \mathrm{~kg} / \mathrm{m}^{2}\right)$, discriminando este último grupo según los diferentes grados de obesidad (I, II, III). En el grupo de hombres con normopeso ( $\mathrm{n}=27)$, $33,3 \%$ presentó PGC en rangos atléticos, 29,6\% PGC en rangos normales y $37 \%$ PGC en rangos de obesidad. En este grupo, el

Tabla 1. Descripción de la población total femenina con las características cineantropométricas generales

\begin{tabular}{|c|c|}
\hline Población femenina & Global \\
\hline $\mathrm{n}$ & 160 \\
\hline Edad (años) (mediana [IQR]) & $41,00[31,00 ; 52,00]$ \\
\hline Talla (cm) (mediana [IQR]) & $160,50[157,00 ; 165,00]$ \\
\hline Peso (kg) (mediana [IQR]) & $69,75[60.53 ; 79,38]$ \\
\hline IMC $\left(\mathrm{kg} / \mathrm{m}^{2}\right)$ (mediana [IQR]) & $26,75[23,90 ; 30,90]$ \\
\hline $\begin{array}{l}\text { Porcentaje de grasa corporal (\%) } \\
\text { (PGC) (mediana [IQR]) }\end{array}$ & $41,80[35,50 ; 47,15]$ \\
\hline $\begin{array}{l}\text { Masa grasa corporal }(\mathrm{kg})(\mathrm{MGC}) \\
\text { (mediana [IQR]) }\end{array}$ & $28,70[22,28 ; 37,02]$ \\
\hline $\begin{array}{l}\text { Área de grasa visceral }\left(\mathrm{cm}^{2}\right)(\mathrm{GV}) \\
\text { (mediana }[\mathrm{IQR}] \text { ) }\end{array}$ & $151,60[104,70 ; 194,45]$ \\
\hline $\begin{array}{l}\text { Masa libre de grasa }(\mathrm{kg}) \text { (MLG) } \\
\text { (mediana [IQR]) }\end{array}$ & $40,85[37,58 ; 44,42]$ \\
\hline $\begin{array}{l}\text { Masa músculo esquelética (Kg) } \\
\text { (MME) (mediana [IQR]) }\end{array}$ & $22,20[20,10 ; 24,20]$ \\
\hline $\begin{array}{l}\text { Tasa metabólica basal (Kcal) } \\
\text { (TMB) (mediana [IQR]) }\end{array}$ & $\begin{array}{c}1252,00[1182,50 ; \\
1329,50]\end{array}$ \\
\hline $\begin{array}{l}\text { Índice cintura cadera (ICC) } \\
\text { (mediana [IQR]) }\end{array}$ & $0,91[0,87 ; 0,96]$ \\
\hline $\begin{array}{l}\text { Perímetro de cuello }(\mathrm{cm})(\mathrm{PCu}) \\
\text { (mediana }[\mathrm{IQR}])\end{array}$ & $35,20[33,20 ; 38,42]$ \\
\hline $\begin{array}{l}\text { Perímetro abdominal (cm) (PA) } \\
\text { (mediana [IQR]) }\end{array}$ & $91,35[83,25 ; 101,98]$ \\
\hline $\begin{array}{l}\text { Perímetro de cadera }(\mathrm{cm})(\mathrm{PCa}) \\
\text { (mediana [IQR]) }\end{array}$ & $99,55[94,80 ; 105,90]$ \\
\hline \multicolumn{2}{|l|}{ PGC.F (\%) } \\
\hline Atlético (14 - 20\%) & $2(1,2)$ \\
\hline Normal (21 - 29\%) & $19(11,9)$ \\
\hline Obeso (>30\%) & $139(86,9)$ \\
\hline $\mathrm{GV}=>100(\%)$ & $124(77,5)$ \\
\hline ICC = >0,86 (\%) & $133(83,1)$ \\
\hline $\mathrm{PA}=>84(\%)$ & $118(73,8)$ \\
\hline $\mathrm{PCu}=>34(\%)$ & $100(62,5)$ \\
\hline
\end{tabular}


Tabla 2. Descripción de la población total femenina con análisis multivariado con relación a IMC, donde se observa el comportamiento cineantropométrico alterado en los pacientes en normopeso y sobrepeso

\begin{tabular}{|c|c|c|c|c|c|c|}
\hline Población femenina & Normal & Sobrepeso & Obesidad I & Obesidad II & Obesidad III & $\mathbf{p}$ \\
\hline $\mathrm{n}$ & 54 & 55 & 40 & 7 & 4 & \\
\hline Edad (años) (mediana [IQR]) & $38,50[30,00,46,75]$ & $46,00[34,50,53,00]$ & $45,00[32,00,53,25]$ & $31,00[30,50,44,50]$ & $45,00[31,50,57,75]$ & 0,321 \\
\hline Talla (cm) (mediana [IQR]) & $162,00[158,00,165,00]$ & $161,00[157,00,164,00]$ & $159,50[156,00,165,00]$ & $159,00[156,00,163,50]$ & $157,50[154,00,162,00]$ & 0,713 \\
\hline Peso (Kg) (mediana [IQR]) & $58,85[54,92,61,65]$ & $69,80[66,00,73,65]$ & $82,10[76,95,86,13]$ & $97,80[93,40,98,55]$ & $116,40[108,83,125,98]$ & $<0,001$ \\
\hline IMC (kg/m²) (mediana [IQR]) & $22,70[21,12,23,90]$ & $26,90[25,95,27,95]$ & $31,95[30,90,33,02]$ & $37,70[36,50,37,90]$ & $43,70[43,48,47,48]$ & $<0,001$ \\
\hline $\begin{array}{l}\text { Porcentaje de grasa corporal } \\
(\%)(\mathrm{PGC}) \text { (mediana [IQR]) }\end{array}$ & $34,00[30,30,37,08]$ & $41,80[38,95,45,00]$ & $47,35[45,40,49,73]$ & $52,30[51,25,52,95$ & $53,90[53,63,54,80]$ & $<0,001$ \\
\hline $\begin{array}{l}\text { Masa grasa corporal (kg) } \\
(\mathrm{MGC})(\text { mediana }[\mathrm{IQR}])\end{array}$ & $20,20[17,12,22,70]$ & $28,60[26,40,31,85]$ & $38,65[38,18,42,20]$ & $50,10[48,55,51,00]$ & $62,35[58,55,68,52]$ & $<0,001$ \\
\hline $\begin{array}{l}\text { Área de grasa visceral }\left(\mathrm{cm}^{2}\right) \\
\text { (GV) (mediana [IQR]) }\end{array}$ & $93,75[72,98,109,55]$ & $151,90[131,00,166,00]$ & $201,10[182,30,211,53]$ & $234,40[211,85,237,20]$ & $233,70[229,42,238,13]$ & $<0,001$ \\
\hline $\begin{array}{l}\text { Masa libre de grasa (kg) (MLG) } \\
\text { (mediana [IQR]) }\end{array}$ & $38,75[36,90,42,42]$ & $40,00[37,45,43,90]$ & $43,15[40,08,45,47]$ & $45,40[43,70,48,45]$ & $54,05[50,27,57,45]$ & $<0,001$ \\
\hline $\begin{array}{l}\text { Masa músculo esquelética (kg) } \\
\text { (MME) (mediana [IQR]) }\end{array}$ & $20,90[19,62,23,03]$ & $21,40[20,05,23,90]$ & $23,80[21,78,25,18]$ & $25,20[23,50,26,65]$ & $30,15[28,03,32,12]$ & $<0,001$ \\
\hline $\begin{array}{l}\text { Tasa metabólica basal (Kcal) } \\
\text { (TMB) (mediana [IQR]) }\end{array}$ & $1207,50[1167,00,1286,25$ & $1233,00[1178,50,1318,00]$ & $1301,50[1235,25,1352,75]$ & $1351,00[1315,00,1417,00]$ & $1537,00[1455,25,1610,75]$ & $<0,001$ \\
\hline $\begin{array}{l}\text { Índice cintura cadera (ICC) } \\
\text { (mediana [IQR]) }\end{array}$ & $0,87[0,84,0,90]$ & $0,92[0,89,0,95]$ & $0,96[0,93,1,00]$ & $0,99[0,91,1,02]$ & $0,91[0,91,0,91]$ & $<0,001$ \\
\hline $\begin{array}{l}\text { Perímetro de cuello }(\mathrm{cm})(\mathrm{PCu}) \\
\text { (mediana [IQR]) }\end{array}$ & $32,55[31,50,33,60]$ & $35,30[34,10,36,35]$ & $38,75[38,20,39,80]$ & $41,90[41,60,43,05]$ & $47,85[46,83,50,05]$ & $<0,001$ \\
\hline $\begin{array}{l}\text { Perímetro abdominal (cm) (PA) } \\
\text { (mediana [IQR]) }\end{array}$ & $80,20[77,70,85,05]$ & $91,50[87,50,95,65]$ & $103,85[98,70,108,32]$ & $114,20[108,30,115,10]$ & $116,75[115,50,120,50]$ & $<0,001$ \\
\hline $\begin{array}{l}\text { Perímetro de cadera }(\mathrm{cm}) \\
(\mathrm{PCa}) \text { (mediana [IQR]) }\end{array}$ & $92,50[90,58,95,12]$ & $99,60[97,85,101,45]$ & $107,25[105,62,109,28]$ & $115,30[113,35,117,70]$ & $128,30[126,85,132,53]$ & $<0,001$ \\
\hline PGC.M (\%) & & & & & & $<0,001$ \\
\hline Atlético (6 - 13\%) & $1(1,9)$ & $1(18)$ & $0(0,0)$ & $0(0,0)$ & $0(0,0)$ & \\
\hline Normal (14 - 24\%) & $19(35,2)$ & $0(0,0)$ & $0(0,0)$ & $0(0,0)$ & $0(0,0)$ & \\
\hline Obeso (> 25\%) & $34(63,0)$ & $54(98,2)$ & $40(100,0)$ & $7(100,0)$ & $4(100,0)$ & \\
\hline GV.M $\geq 100 \mathrm{~cm}^{2}(\%)$ & $22(40,7)$ & $51(92,7)$ & $40(100,0)$ & $7(100,0)$ & $4(100,0)$ & $<0,001$ \\
\hline ICC.M $\geq 0,90(\%)$ & $34(63,0)$ & $51(92,7)$ & $39(97,5)$ & $5(71,4)$ & $4(100,0)$ & $<0,001$ \\
\hline PA.M $\geq 92 \mathrm{~cm}(\%)$ & $16(29,6)$ & $51(92,7)$ & $40(100,0)$ & $7(100,0)$ & $4(100,0)$ & $<0,001$ \\
\hline PCu.M $\geq 37$ CM (\%) & $8(14,8)$ & $41(74,5)$ & $40(100,0)$ & $7(100,0)$ & $4(100,0)$ & $<0,001$ \\
\hline
\end{tabular}

$11,1 \%$ presentó grasa visceral en rangos elevados $\left(>100 \mathrm{~cm}^{2}\right)$, el PA anormal se observó en 18,5\%, PCu >38 cm se encontró en $25,9 \%$ e ICC $>0,91$, fue de $25,9 \%$. En el grupo de sobrepeso $(n=35), 17,1 \%$ de ellos presentó PGC en rangos normales y $82,9 \%$ PGC en rangos de obesidad, 42,9\% grasa visceral mayor de $100 \mathrm{~cm}^{2}$; el PA anormal se observó en $65,7 \%$, PCu $>38 \mathrm{~cm}$ se encontró en $71,4 \%$ e ICC $>0,91$, fue de $57,1 \%$. En el grupo de obesidad ( $\mathrm{n}=35)$, el $100 \%$ presentó PGC en rangos de obesidad, $100 \%$ grasa visceral mayor de $100 \mathrm{~cm}^{2}$, el PA anormal se observó en $100 \%, \mathrm{PCu}>38 \mathrm{~cm}$ se encontró en 100\% (aunque en el grupo de obesidad grado I, $92 \%$ de la población) y de ICC $>0,91$, fue de $92 \%$.
En el grupo de mujeres con normopeso ( $n=54), 1,9 \%$ de ellas presentó PGC en rangos atléticos, 35,2\% PGC en rangos normales y $63 \%$ PGC en rangos de obesidad; $40,7 \%$ grasa visceral mayor de $100 \mathrm{~cm}^{2}$. El PA anormal se observó en 29,6\%, $\mathrm{PCu}>34 \mathrm{~cm}$ se encontró en 14,8\% y el ICC >0,85 fue de $63 \%$. En el grupo de sobrepeso ( $\mathrm{n}=55), 1,8 \%$ presentó PGC en rango atlético, mientras el 98,2\% PCG en rangos de obesidad; 92,7\% grasa visceral mayor de $100 \mathrm{~cm}^{2}$. El PA anormal se observó en 92,7\%; PCu >34 cm, se encontró en 74,5\% y el ICC >0,85 fue de $92,7 \%$. En el grupo de obesidad $(n=51)$, el $100 \%$ presentó PGC en rangos de obesidad y $100 \%$ grasa visceral mayor de 100 $\mathrm{cm}^{2}$. El PA anormal se observó en $100 \%$ al igual que $\mathrm{PCu}$, de 
Tabla 3. Descripción de la población total masculina con las características cineantropométricas generales

Población masculina

\begin{tabular}{l|c}
\multicolumn{1}{c|}{$\mathrm{n}$} & 97 \\
\hline Edad (años) (mediana [IQR]) & $43,42(14,42)$ \\
\hline Talla $(\mathrm{cm})$ (mediana [IQR]) & $171,67(6,43)$ \\
\hline Peso $(\mathrm{kg})$ (mediana [IQR]) & $83,00[73,20 ; 92,90]$ \\
\hline IMC (kg/m²) (mediana [IQR]) & $28,00[24,70 ; 31,80]$ \\
\hline $\begin{array}{l}\text { Porcentaje de grasa corporal }(\%) \\
\text { (PGC) (mediana [IQR]) }\end{array}$ & $30,62(8,84)$ \\
\hline
\end{tabular}

Masa grasa corporal (kg) (MGC) (mediana [IQR])

\begin{tabular}{l|c}
\hline $\begin{array}{l}\text { Área de grasa visceral }\left(\mathrm{cm}^{2}\right)(\mathrm{GV}) \\
\text { (mediana [IQR]) }\end{array}$ & $102,60[80,10 ; 159,90]$ \\
\hline $\begin{array}{l}\text { Masa libre de grasa (kg) (MLG) } \\
\text { (mediana [IQR]) }\end{array}$ & $32,72(7,45)$ \\
\hline $\begin{array}{l}\text { Masa músculo esquelética (kg) } \\
\text { (MME) (mediana [IQR]) }\end{array}$ & $1616,81(161,04)$ \\
\hline $\begin{array}{l}\text { Tasa metabólica basal (Kcal) (TMB) } \\
\text { (mediana [IQR]) }\end{array}$ & $0,94(0,08)$ \\
\hline $\begin{array}{l}\text { Índice cintura cadera (ICC) (mediana } \\
\text { [IQR]) }\end{array}$ & $38,20[36,60 ; 40,10]$ \\
\hline $\begin{array}{l}\text { Perímetro de cuello (cm) (PCu) } \\
\text { (mediana [IQR]) }\end{array}$ & $98,62(14,62)$ \\
\hline $\begin{array}{l}\text { Perímetro abdominal (cm) (PA) } \\
\text { (mediana [IQR]) }\end{array}$ & $104,00[98,40 ; 109,30]$ \\
\hline $\begin{array}{l}\text { Perímetro de cadera (cm) (PCa) } \\
\text { (mediana [IQR]) }\end{array}$ & $9(9,3)$ \\
\hline PGC.M (\%) & $14(14,4)$ \\
\hline Atlético (6 - 13\%) & $74(76,3)$ \\
\hline Normal (14 - 24\%) & $53(54,6)$ \\
\hline Obeso (>25\%) & $59(60,8)$ \\
\hline GV.M $\geq 100 \mathrm{~cm}{ }^{2}(\%)$ & $63(64,9)$ \\
\hline ICC.M $\geq 0,90(\%)$ & $65,0)$ \\
\hline PA.M $\geq 92 \mathrm{~cm}(\%)$ & \\
\hline PCu.M $\geq 37 \mathrm{~cm}(\%)$ & \\
\hline & \\
\hline
\end{tabular}

forma interesante se observó que en el ICC $>0,85$, se presentaron diferencias según categorías de obesidad, grado I, grado II y grado III, mostrando alteración del 97,5\%, 71,5\% y 100\%, respectivamente; al hacer correlación con antecedentes quirúrgicos, se encontraron procedimientos estéticos asociados.

\section{Discusión}

El IMC, ideado por el estadístico belga Adolphe Quetelet, es una razón matemática que asocia la masa y la talla de un individuo, con un impacto como herramienta de medida de obesi- dad. Como se había mencionado, no permite diferenciar entre peso asociado a músculo o a grasa ${ }^{(2)}$. Como resultado, la relación entre el IMC y el contenido de grasa corporal varía de acuerdo con la constitución y la proporción corporal, demostrándose repetidamente que un IMC determinado puede no corresponder al mismo grado de obesidad entre las poblaciones ${ }^{(1)}$. Ya se ha determinado que hay otras medidas antropométricas que tienen mejor correlación con morbimortalidad como lo son índice cintura-altura (ICA), índice cintura-cadera (ICC), perímetro abdominal (PA) y perímetro de cuello (PCu) ${ }^{(21,22)}$.

Al poder caracterizar esta muestra según las diferentes medidas cineantropométricas mencionadas, podemos evidenciar, como en múltiples estudios, que el IMC no es mal indicador de obesidad, y de allí se ha acuñado el término "adiposopatía", en donde se reúne el cuadro de alteraciones del tejido graso, que aunque no tenga relación con IMC, sí explica el advenimiento de ECNT (enfermedades crónicas no transmisibles), en población en sobrepeso e incluso en normopeso.

De igual forma, en el análisis de los datos de esta cohorte podemos observar cómo hay una relación directa entre niveles PGC, GV y medidas antropométricas con los diferentes grados de obesidad, según IMC. Sin embargo, en el grupo de normopeso y sobrepeso se aprecia población con alto PGC y GV en ambos grupos, tanto de hombres como de mujeres, con medidas antropométricas elevadas que confieren un riesgo cardiometabólico específico.

Es importante destacar en la población femenina con IMC normal, que el valor de ICC es comparativamente mayor que en hombres, seguido por relación muy homogénea entre los valores de PGC y $\mathrm{PCu}$ y menos relevante con PA, sugiriendo que el PA no es la medida más adecuada en este grupo, puesto que podría verse alterada por características fenotípicas propias de la población femenina, intervenciones quirúrgicas o la relación con el contenido de GV; sin embargo, esto no se ve en la población masculina en la misma categoría de IMC.

En cuanto al grupo de sobrepeso, desde el punto de vista de riesgo cardiometabólico, esta población se podría considerar la más vulnerable, dado su valor numérico alto en todas las variables, que infieren un alto riesgo antropométrico, y que se relacionan con un elevado PGC y GV; se puede observar este patrón tanto en hombres como en mujeres, siendo considerablemente más dramático en mujeres; por tanto, en este grupo se podría considerar orientar medidas destinadas a determinar el riesgo individual, más allá de la toma de peso y talla, y también relacionarlo con PGC, ICC, PA, PCu, haciendo la salvedad de que no es necesario disponer de tecnologías costosas para este fin, pues la correlación de los índices y la estimación matemática de PGC por BAI o por CUN-BAE ${ }^{(16)}$, permitiría hacer una aproximación adecuada para poder abordar múltiples factores de riesgo.

Por último, es también importante mencionar que la composición corporal en programas destinados al manejo de al- 
Tabla 4. Descripción de la población total masculina con análisis multivariado con relación a IMC, donde se observa el comportamiento cineantropométrico alterado en los pacientes en normopeso y sobrepeso

\begin{tabular}{|c|c|c|c|c|c|c|}
\hline Población masculina & Normal & Sobrepeso & Obesidad I & Obesidad II & Obesidad III & $\mathbf{p}$ \\
\hline $\mathrm{n}$ & 27 & 35 & 25 & 6 & 4 & \\
\hline Edad (años) (mediana [IQR]) & $41,04(15,08)$ & $43,12(12,07)$ & $47,51(16,52)$ & $38,00(13,37)$ & $44,75(17,17)$ & 0,492 \\
\hline Talla $(\mathrm{cm})$ (mediana [IQR]) & $171,50(5,67)$ & $171,31(6,76)$ & $171,56(7,05)$ & $173,75(6,00)$ & $173,50(7,42)$ & 0,901 \\
\hline Peso (Kg) (mediana [IQR]) & $69,80[62,90,73,85]$ & $82,50[76,25,84,90]$ & $93,70[86,60,100,40]$ & $111,35[108,65,114,65]$ & $131,40[121,95,140,45]$ & $<0,001$ \\
\hline IMC $\left(\mathrm{kg} / \mathrm{m}^{2}\right)$ (mediana [IQR]) & $23,80[22,05,24,25]$ & $27,10[26,40,28,80]$ & $32,20[31,00,32,90]$ & $36,40[36,32,36,48]$ & $44,15[42,75,44,92]$ & $<0,001$ \\
\hline $\begin{array}{l}\text { Porcentaje de grasa corporal } \\
(\%)(P G C) \text { (mediana [IQR]) }\end{array}$ & $22,24(6,75)$ & $28,82(4,78)$ & $36,50(3,96)$ & $42,62(5,24)$ & $48,23(3,77)$ & $<0,001$ \\
\hline $\begin{array}{l}\text { Masa grasa corporal (kg) } \\
\text { (MGC) (mediana [IQR]) }\end{array}$ & $15,70[10,90,18,90]$ & $22,60[19,60,25,85]$ & $34,60[31,80,36,90]$ & $46,10[42,95,51,20]$ & $63,15[59,68,66,70]$ & $<0,001$ \\
\hline $\begin{array}{l}\text { Área de grasa visceral }\left(\mathrm{cm}^{2}\right) \\
\text { (GV) (mediana [IQR]) }\end{array}$ & $63,30[45,50,80,45]$ & $94,40[81,50,108,35]$ & $158,50[139,90,179,90]$ & $212,45[191,55,237,85]$ & $238,90[228,25,253,05]$ & $<0,001$ \\
\hline $\begin{array}{l}\text { Masa libre de grasa (kg) (MLG) } \\
\text { (mediana [IQR]) }\end{array}$ & $53,03(6,06)$ & $57,38(6,09)$ & $60,28(7,44)$ & $63,45(7,56)$ & $67,78(6,94)$ & $<0,001$ \\
\hline $\begin{array}{l}\text { Masa músculo esquelética (kg) } \\
\text { (MME) (mediana [IQR]) }\end{array}$ & $29,72(3,71)$ & $32,32(3,67)$ & $33,92(4,45)$ & $35,92(4,41)$ & $38,23(4,37)$ & $<0,001$ \\
\hline $\begin{array}{l}\text { Tasa metabólica basal (Kcal) } \\
\text { (TMB) (mediana [IQR]) }\end{array}$ & $1515,52(130,94)$ & $1609,54(131,52)$ & $1671,88(160,70)$ & $1740,50(162,75)$ & $1834,50(149,81)$ & $<0,001$ \\
\hline $\begin{array}{l}\text { Índice cintura cadera (ICC) } \\
\text { (mediana [IQR]) }\end{array}$ & $0,88(0,06)$ & $0,92(0,05)$ & $1,00(0,06)$ & $1,02(0,05)$ & $0,99(0,10)$ & $<0,001$ \\
\hline $\begin{array}{l}\text { Perímetro de cuello }(\mathrm{cm})(\mathrm{PCu}) \\
\text { (mediana [IQR]) }\end{array}$ & $36,10[35,15,37,05]$ & $37,80[37,00,38,80]$ & $40,10[39,00,41,10]$ & $44,25[42,92,45,58]$ & $50,55[48,23,52,78]$ & $<0,001$ \\
\hline $\begin{array}{l}\text { Perímetro abdominal }(\mathrm{cm})(\mathrm{PA}) \\
\text { (mediana [IQR]) }\end{array}$ & $83,91(7,42)$ & $95,01(6,10)$ & $109,54(7,87)$ & $121,05(5,58)$ & $127,58(13,29)$ & $<0,001$ \\
\hline $\begin{array}{l}\text { Perímetro de cadera }(\mathrm{cm}) \\
(\mathrm{PCa}) \text { (mediana [IQR]) }\end{array}$ & $96,20[93,40,98,20]$ & $103,50[100,70,105,15]$ & $109,90[107,50,111,60]$ & $118,00[117,53,118,62]$ & $129,35[126,35,132,00]$ & $<0,001$ \\
\hline PGC.M (\%) & & & & & & $<0,001$ \\
\hline Atlético (6 - 13\%) & $9(33,3)$ & $0(0,0)$ & $0(0,00)$ & $0(0,00)$ & $0(0,00)$ & \\
\hline Normal (14 - 24\%) & $8(29,6)$ & $6(17,1)$ & $0(0,00)$ & $0(0,00)$ & $0(0,00)$ & \\
\hline Obeso (> 25\%) & $10(37,0)$ & $29(82,9)$ & $25(100,0)$ & $6(100,0)$ & $4(100,0)$ & \\
\hline GV.M $\geq 100 \mathrm{~cm}^{2}(\%)$ & $3(11,1)$ & $15(42,9)$ & $25(100,0)$ & $6(100,0)$ & $4(100,0)$ & $<0,001$ \\
\hline ICC.M $\geq 0,90(\%)$ & $7(25,9)$ & $20(57,1)$ & $23(92,0)$ & $6(100,0)$ & $3(75,0)$ & $<0,001$ \\
\hline PA.M $\geq 92 \mathrm{~cm}(\%)$ & $5(18,5)$ & $23(65,7)$ & $25(100,0)$ & $6(100,0)$ & $4(100,0)$ & $<0,001$ \\
\hline PCu.M $\geq 37$ CM (\%) & $7(25,9)$ & $25(71,4)$ & $23(92,0)$ & $6(100,0)$ & $4(100,0)$ & $<0,001$ \\
\hline
\end{tabular}

teraciones de peso, el IMC no es el mejor indicador de seguimiento, pues como vimos hay muchas variables que toman mayor relevancia más allá de la relación talla y peso, que no necesariamente debe cambiar cuando se buscan objetivos específicos según composición corporal.

Por lo anterior, podemos concluir que la composición corporal junto a la medición de índices antropométricos tiene una relevancia mayor al momento de determinar riesgo del paciente, objetivos a alcanzar y seguimiento del proceso, los cuales deben ser asociados al IMC, evitando que este último sea nuestro único indicador de éxito.

De igual forma, en un país donde la obesidad y sobrepeso se encuentra en crecimiento con una prevalencia de sobrepe- so mayor del $50 \%$, existe la necesidad de determinar el real riesgo cardiometabólico, puesto que esta es la población más subestimada, al no tener una continua atención o promoción de hábitos nutricionales y deportivos adecuados.

Se podría considerar que los biotopos establecidos por medición de PGC se presentan de igual forma en nuestra población, donde se especifica un biotipo A con bajo nivel de PGC y GV el cual es atlético, de bajo riesgo, el biotipo B, con elevación de PGC y VG con medidas antropométricas limítrofes y, por último, un biotipo $\mathrm{C}$, donde se encuentran alteradas tanto PGC, VG como las medidas antropométricas; este biotipo es de alto riesgo y se presenta de forma independiente al IMC. 


\section{Conclusión}

Podemos concluir que la composición corporal junto a la medición de índices antropométricos tiene una relevancia mayor al momento de determinar el riesgo del paciente, objetivos a alcanzar y seguimiento del proceso, los cuales deben ser asociados al IMC, evitando que este último sea nuestro único indicador de éxito.

De igual forma, en un país donde la obesidad y sobrepeso se encuentran en crecimiento con una prevalencia de sobrepeso mayor del 50\%, existe la necesidad de determinar el riesgo cardiometabólico real puesto que ésta es la población más subestimada, al no tener una continua aten- ción o promoción de hábitos nutricionales y deportivos adecuados.

Es pertinente aclarar que necesitamos un tamaño poblacional mayor para poder establecer la caracterización global en nuestra región, así como continuar con más estudios que demuestren la correlación de estas variables antropométricas con variables bioquímicas.

\section{Conflictos de interés}

$\mathrm{Ni}$ el estudio, ni los autores recibieron patrocinio de la industria farmacéutica o de sectores académicos o gubernamentales, previo o durante la investigación del estudio.

\section{Referencias}

1. Craig PL. Body composition differences between Polynesians and Caucasians assessed by bioelectrical impedance Differences in body composition between Tongans and Australians : time to rethink the healthy weight ranges? 2014.

2. World Health Organization. Obesity: Preventing and managing the global epidemic. Report of a WHO Consultation 2004.

3. Haslam DW, James WP, Obesity. Lancet 2005; 366: 1197-1209.

4. Hamann A. Obesity Update 2017. Diabetologe [Internet] 2017; 13(5): 33141. Available from: www.oecd.org/health/obesity-update.htm

5. ICBF. Encuesta Nacional de la Situación Nutricional 2015; 1-58. Available from: http://www.culturarecreacionydeporte.gov.co/sites/default/ files/2015_diseno-general-ensin_.pdf

6. Abdelaal M, le Roux CW, Docherty NG. Morbidity and mortality associated with obesity. Ann Transl Med [Internet] 2017; 5(7):161. Available from: http://atm.amegroups.com/article/view/14394/14547

7. Shuval K, Barlow CE, Finley CE, Gabriel KP, Schmidt MD, Defina LF. Standing obesity, and metabolic syndrome findings from the cooper center longitudinal study. Mayo Clin Proc. 2015; 90(11): 1524-32.

8. Frühbeck G, Diez-Caballero A, Gómez-Ambrosi J, Cienfuegos JA, et al. Preventing obesity. Doctors underestimate obesity. BMJ 2003; 326(7380): 102-3.

9. Frühbeck G. Screening and interventions for obesity in adults. Ann Intern Med. 2004 Aug 3;141(3):245-6

10. Wildman RP, Muntner P, Reynolds K, Mcginn AP. The Obese Without Cardiometabolic Risk Factor Clustering and the Normal Weight With Cardiometabolic Risk Factor Clustering. Arch Intern Med. 2013; 168(15): 1617-24.

11. Frühbeck G. Screening and interventions for obesity in adults. Ann Intern Med 2004; 141: 245-246; author reply 246

12. Das SK. Body composition measurement in severe obesity. Curr Opin Clin Nutr Metab Care. 2005; 8: 602-606

13. Fields DA, Goran MI, McCrory MA. Body- composition assessment via airdisplacement plethysmography in adults and children: a review. Am J Clin Nutr. 2002; 75: 453- 467 .
14. Ginde SR, Geliebter A, Rubiano F, et al. 14. Air displacement plethysmography: vali- dation in overweight and obese subjects. Obes Res. 2005; 13 1232-1237.

15. Heo M, Faith MS, Pietrobelli A, Heymsfield SB. Percentage of body fat cutoffs by sex, age, and race-ethnicity in the US adult population from NHANES 1999-2004. 2012; 594-602.

16. Javier Gómez-Ambrosi, Camilo Silva, Victoria Catalán, Amaia Rodríguez Juan Carlos Galofré, Javier Escalada, Victor Valentí, et al. Clinical Usefulness of a New Equation for Estimating Body Fat. Blood Press. 2012; 1-6.

17. Catalán V, Gómez-Ambrosi J, Ramirez B, Rotellar F, Pastor C, Silva C, et al Proinflammatory cytokines in obesity: Impact of type 2 diabetes mellitus and gastric bypass. Obes Surg. 2007; 17(11): 1464-74.

18. Catalán V, Gómez-Ambrosi J, Rodríguez A, Ramírez B, Rotellar F, Valentí V, et al. Increased circulating and visceral adipose tissue expression levels of YKL-40 in obesity-associated type 2 diabetes are related to inflammation: impact of conventional weight loss and gastric bypass. J Clin Endocrinol Metab. 2011; 96(1): 200-9.

19. Gallo J, Ochoa J, Balparda J, Aristizábal D. Puntos de corte del perímetro de la cintura para identificar sujetos con resistencia a la insulina en una población colombiana. Acta Med Colomb [Internet] 2013; 38: 118-26. Available from: http://www.scielo.org.co/pdf/amc/v38n3/v38n3a06.pdf

20. Jennifer Linge, Magnus Borga, Janne West, Theresa Tuthill, Melissa R. Miller, Alexandra Dumitriu, et al. Body Composition Profiling in the UK Biobank Imaging Study. Obesity Journal. 2018. doi:10.1002/oby.22210

21. El-Din ASHNE-MSA-TM. Neck circumference as a simple screening measure. Maced J Med Sci. 2013; 6(3): 232-7.

22. WHO, Development $\mathrm{D}$ of $\mathrm{N}$ for $\mathrm{H}$ and. Waist circumference and waist-hip ratio: report of a WHO expert consultation 2008; 8: 8-11.

23. Schneider HJ, Friedrich N, Klotsche J, Pieper L, Nauck M, John U, et al. The predictive value of different measures of obesity for incident cardiovascular events and mortality. J Clin Endocrinol Metab. 2010; 95(4): 1777-85. 